\title{
Studies of catalysts and catalytic reactions by infrared thermography
}

by MARENGO S. and COMOTTI P.

\author{
Stazlone Sperimentale per i Combustibili, viale A. De Gasperi 3 \\ jo097 S. Donato Milanese, Italy
}

\begin{abstract}
This communication shows that infrared thermography can be a valuable technique for catalyst characterization and for the study of catalytic processes. The possibility of drawing detailed thermal maps of catalytic systems under working conditions, associated with the growing availability of image inilysis methods, allows a range of applications in the study of acid- base properties of solid materials and in the description of thermal effects produced in chemical reactors by exothermic catalytic reactions.
\end{abstract}

\section{Introduction}

Infrared thermography is utilized by the chemical industry mainly in large-scale applications, as for instance in energy saving and in routine inspection of catalytic reactors, procoss lines, valves and other units. Only few examples can be found in the literature concorning the utilization of infrared thermography in catalysis on the bench scale [1-4].

Aim of this communication is to report on some recent applications of the technique in the fleld of catalysis, which may be of interest for the chemical industry. The measurement of acidbase properties of solid materials utilized as heterogeneous catalysts will be described. The posilbility of detailed investigation on the thermal effects produced in chemical reactors by exothermic reactions will also be addressed.

\section{1. xporimental}

Two thermographic apparatuses were utilized: in early experiments, an AGA Thermovision 102 equipped with a Dyscon system for real-time conversion of black/white images into 10colour pictures; in the latest studies of catalytic reactions, a state-of-the-art AGEMA Thermovision 900 apparatus operating in the wavelength range 2-5.4 microns. The sample, in Ilie form of single pellet or of fine particles, was placed in a specially designed tubular reactor Vansparent to IR radiation and exposed to a flow of chemical reactant in the gaseous or liquid phiase at atmospheric pressure.

\section{5 tudy of acid-base properties of solids}

The determination of acid-base properties of materials utilized as catalysts or supports was based on the measurement of the thermal effect produced by adsorption of basic $\mathrm{NH}_{3}$, Allylamine) or acidic $\left(\mathrm{CO}_{2}\right)$ compounds.

Proliminary experiments showed that the signal obtained upon adsorption of these molecules on the sample at room temperature, exceeded by 1 to 2 orders of magnitude the onenglivity of the thermographic system $(0.1 \mathrm{~K})$. In addition to this good response, a relatively high spatial resolution of $0.1 \mathrm{~mm}$ was achieved by utilizing a lens for close-up work. This sillowod a detailed study of such small samples as single catalyst particles in the form of pellets Willi diameter of 1 to $3 \mathrm{~mm}$. 
When these particles were exposed to a continuous flow of gas containing a basic compound, the acid sites on the surface were visualized by an increase in temperature due to the adsorption of the base; the difference in acidity of the samples could be easily evaluated. In addition, this technique offers the possibility of examining the homogeneity of distribution of the active sites on the surface of a single pellet, thus providing useful information on the production process.

The quantitative determination of acidic and basic sites in solid materials is a difficult analysis, which is time consuming and, in the case of colored indicators, presents many limitations as for the nature of the sample.

Our experiments showed that this measurement can be easily performed by infrared thermography. The method offers also the advantage of testing the catalysts in a tubular flow reactor, under conditions similar to those of their practical use. Other advantages are the rapidity of the measurement, the relatively simple experimental setup, the non-destructive nature of the test. For the measurement of acid or basic sites, the sample in the form of fine particles was placed in a vertical reactor and exposed to a controlled flow of gas containing a known amount of acid or base. By plotting the maximum of the thermal effect vs. the amount of the base added, a titration curve is obtained (Fig.1). The maximum of this curve can be taken as a measure of the average acid strength of the sample, and the amount of base corresponding to this maximum is related to total acidity. The titration curves show the presence of strong acid sites in the heteropolyacid, whereas the zeolite sample exhibits a larger amount of surface sites of intermediate acidity.

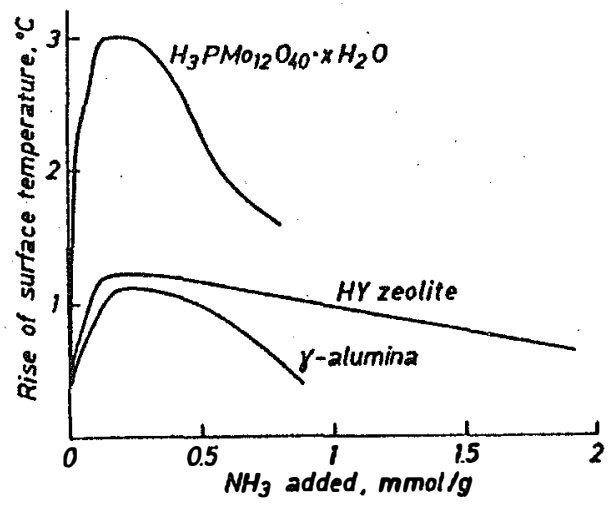

Fig. 1. Thermographic titration of solid acids.

\section{Thermal effects in a two-phase reactor}

The hydrogenation of ethylene was utilized as a model reaction for the study of the thermal effects in a two-phase reactor. A vertical tube was charged with $1 \mathrm{~g}$ of $2 \% \mathrm{Rh} / \mathrm{Al}_{2} \mathrm{O}_{3}$ in the form of fine particles $(0.1-0.3 \mathrm{~mm})$. Ethylene, helium and hydrogen were fed upward at room temperature.

At start up, a heat front forms in the initial part of the catalyst bed. This front, generated by the exothermic reaction in quasi-adiabatic conditions, corresponds to the reacting portion of the catalyst. In the experimental conditions of the test, due to the poor heat transfer properties of the gas phase, the heat generated by the reaction kept accumulating in the initial portion of the catalyst bed, where the self-sustained reaction reached a high rate. As $100 \%$ of the feed was converted in this region, most of the remaining catalyst bed was substantially inactive (Fig. 2). The thermal map of the catalyst remained almost unchanged over a wide range of reactant feed rates and temperatures. 
http://dx.doi.org/10.21611/qirt.1994.002
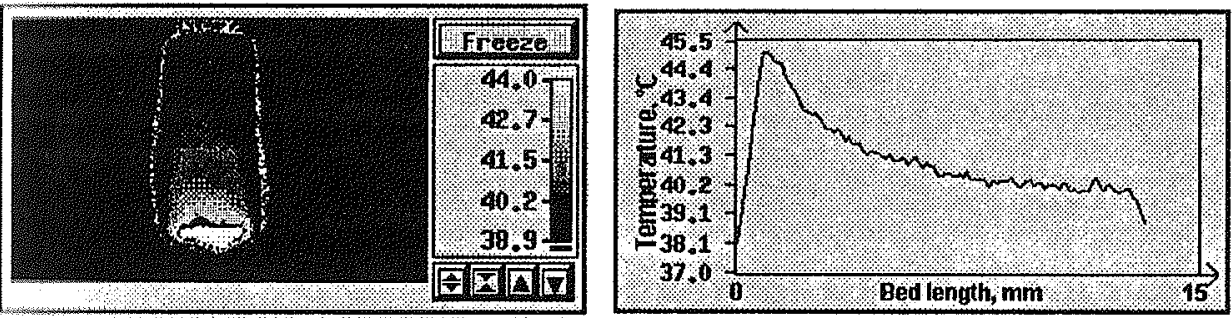

Fig. 2. Thermal map and temperature profile of a reacting catalyst bed.

The heat (reaction) front was stable; however, unique dynamic effects were observed when a small amount of $\mathrm{CO}(1 \%)$ was added to the feed. The front propagated along the bed in the direction of the gas flow as a consequence of the gradual inhibition of the active sites caused by adsorption of $\mathrm{CO}$. The rate of propagation of the reaction front was about two orders of magnitude lower than the superficial velocity of the gas phase. When CO was removed, the reaction front moved back against the gas flow, and eventually reached the initial position. The image processing facility allowed monitoring and studying of this phenomenon in real time.

\section{i. Three-phase reactors}

Three-phase reactors are widely utilized in the chemical industry, mainly in petroleum refining. The description of the heat and mass transfer phenomena in these reaction systems is quite difficult, albeit of great importance for reactor modeling and process scale up. Our preliminary experiments suggest that infrared thermography offers the possibility of getting a deeper in mapping of the surface of the catalyst bed recorded under reaction conditions.

Cyclohexene hydrogenation was utilized as a model reaction in the study of a trickle-bed feactor. The catalyst $\left(0.5 \% \mathrm{Rh} \mathrm{Al}_{2} \mathrm{O}_{3}\right.$ in the form of pellets with diam. of $\left.3 \mathrm{~mm}\right)$ was exposed to a downward flow of $\mathrm{H}_{2}$ and cyclohexene at $25-50^{\circ} \mathrm{C}$, with LHSV ranging from 60 to $240 \mathrm{~h}^{-1}$. The infrared image of the catalyst bed showed formation of hot spots with peak temperatures of about $40^{\circ} \mathrm{C}$, corresponding to zones only partially wetted, in which a gas-solid reaction took place. Fig. 3 shows the thermal profile of the reacting bed, recorded along a vertical line.

The analysis of this kind of data can provide a detailed description of some basic phenomena associated with three-phase reactors, such as catalyst wetting, bubbling, ogcurrence of gas phase reaction.

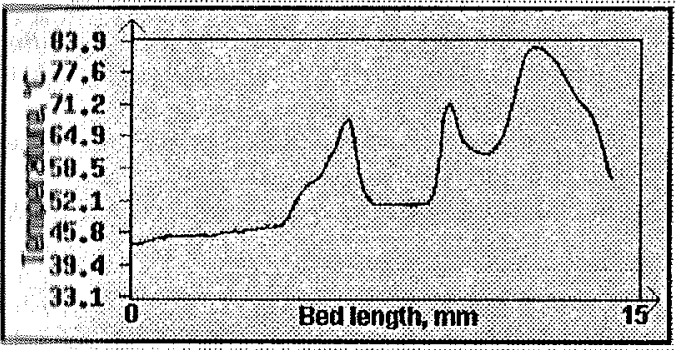

la. 3. Thermal profile of a trickle bed. 
http://dx.doi.org/10.21611/qirt.1994.002

\section{Acknowledgments}

The authors thank AGEMA srl, Italy, for technical assistance and Mr. S. Scappatura for a valuable contribution in performing the experiments.

\section{REFERENCES}

[1] PAWLICKI (P.C.) and SCHMITZ (R.A.), CHEM. Eng. Prog. 83(2),(1987)40-45.

[2] KELLOW (J.) and WOLF (E.E.), Catalysis Today, 9(1991)47-51.

[3] CARIMATI (A.),MARENGO (S.) and RAIMONDINI (G.), Proceedings of the 11th Canadian Symposium on Catalysis, (J. Monnier Ed.), Halifax, Canada, 1990, pp. 143-151.

[4] MARENGO (S.), RAIMONDINI (G.) and COMOTTI (P.), in GUCZI (L.) (Editor),"New Frontiers in Catalysis", Elsevier, Amsterdam, 1993, Part C, p. 2573. 